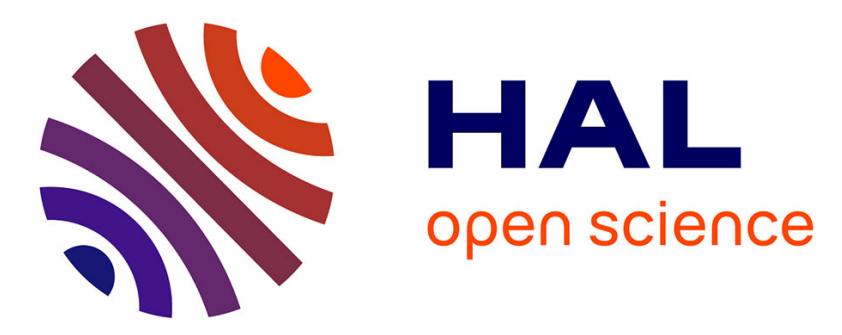

\title{
Robust PI observer design for hybrid heterogeneous multiple models with delayed measurements
}

\author{
Rodolfo Orjuela, Benoît Marx, José Ragot, Didier Maquin
}

\section{To cite this version:}

Rodolfo Orjuela, Benoît Marx, José Ragot, Didier Maquin. Robust PI observer design for hybrid heterogeneous multiple models with delayed measurements. 3rd IFAC Conference on Analysis and Design of Hybrid Systems, ADHS'09, Sep 2009, Zaragoza, Spain. pp.CDROM, 10.3182/20090916-3ES-3003.00068 . hal-00402428

\section{HAL Id: hal-00402428 https://hal.science/hal-00402428}

Submitted on 31 Mar 2014

HAL is a multi-disciplinary open access archive for the deposit and dissemination of scientific research documents, whether they are published or not. The documents may come from teaching and research institutions in France or abroad, or from public or private research centers.
L'archive ouverte pluridisciplinaire HAL, est destinée au dépôt et à la diffusion de documents scientifiques de niveau recherche, publiés ou non, émanant des établissements d'enseignement et de recherche français ou étrangers, des laboratoires publics ou privés. 


\title{
Robust PI observer design for hybrid heterogeneous multiple models with delayed measurements
}

\author{
Rodolfo Orjuela, Benoît Marx, José Ragot \\ and Didier Maquin \\ Centre de Recherche en Automatique de Nancy \\ UMR 7039, Nancy-Université, CNRS \\ 2, Avenue de la Forêt de Haye \\ 54516 Vandouvre-lès-Nancy, France
}

\begin{abstract}
This paper deals with the problem of state estimation for hybrid systems modelled via multiple models and subject to delayed measurements. In contrast to the most popular results found in the multiple model literature, we consider heterogeneous multiple models, also known as decoupled multiple model, which allow to use submodels whose state space dimension can be different. On the basis of this multiple model a proportional-integral observer (PIO) is designed in order to cope with the robust state estimation with respect to disturbances. Sufficient delay-independent conditions for ensuring robust performances (attenuation level) and dynamic performances (exponential convergence) of the estimation error are provided in terms of LMIs using the Lyapunov-Krasovskii method. The validity of the proposed methodology is illustrated by an academic example.
\end{abstract}

Keywords: heterogeneous multiple models; state estimation; proportional-integral observer; delayed measurements

\section{INTRODUCTION}

Context. Time-delay or dead time is frequently encountered in several real-world processes, such as chemical and thermal processes, electrical and communications networks, etc. Indeed, any system has an amount of time, more or less important, between its expected response and its actual response. In many industrial applications time-delays must unavoidably be considered as an inherent part of the system, and consequently, they must be accounted for in the mathematical model of the system under investigation [Smith and Corripio, 1985]. Therefore, considerable attention has been paid in the past decades to systems with delays and appropriate theoretical tools, in the frequency and in the time domain, for the analysis, control and state estimation are well established [Bliman, 2001, Sename, 2001, Richard, 2003]. Among them, the well known Lyapunov-Krasovskii functional method is largely adopted for stability analysis of linear or nonlinear delayed systems. This method provides generally sufficient conditions in a LMI form which can be solved in polynomialtime using appropriated standard convex optimization algorithms [Boyd et al., 1994].

On the other hand, complex industrial systems generally present a nonlinear dynamic behaviour in particular when the whole operating range (global behaviour) must be considered instead of a reduced operating range in the neighbourhood of an operating point (local behaviour). This situation is commonly encountered in the state estimation problems because all admissible trajectories of the system must be taken into consideration. Hence, adequate nonlinear model representations must be used to cope effectively with the global modelling of these systems. A number of quite diverse similar modelling frameworks such as hybrid systems, switched systems, multiple models, etc. are proposed to dealing with the modelling of systems that evolves according to different dynamics in the operating space.

Tools and proposed method. The nonlinear behaviour of a system can accurately be represented using the multiple model approach [Murray-Smith and Johansen, 1997, Boukhris et al., 1999]. Multiple models have been recognized as a popular and a powerful modelling tool of complex nonlinear systems. In this approach the operating space of the system under investigation is decomposed into a number of operating zones, each of them being characterised by a submodel of reduced dimension. The interpolation of submodels with the help of weighting functions, associated to each operating zone, allows the global representation of the nonlinear system. Let us notice finally that the basis of the multiple model approach can be easily related to the hybrid system philosophy in which the interaction of a set of submodels changes according to a dynamic law. Indeed, smooth as well as crisp (switching) transitions between the submodels can be considered for more flexibility in modelling stage.

At this point, it is important to point out that the interpolation of submodels can be operated using many schemas [Filev, 1991, Gregorcic and Lightbody, 2008], among these realisations two schemas can be distinguished: via an interpolation of parameters (e.g. Piecewise Affine Systems 
or Takagi-Sugeno multiple model) or via an interpolation of outputs (e.g. decoupled multiple model). Many contributions concern the analysis, the control and the state estimation of the first multiple model realisation (with or without delays) [Murray-Smith and Johansen, 1997, Babuska, 1998, Tanaka and Wang, 2001, Lin et al., 2007], much less are devoted to observer design using decoupled multiple models. In this work, the latter realisation will be employed for dealing with the problem of state estimation for nonlinear hybrid systems with delayed measurements as an extension of the ideas proposed previously [Orjuela et al., 2007, 2008].

Related work. Uppal et al. [2006] have shown a successful implementation of this structure in order to cope with the state estimation for fault detection and isolation. However, only a note of the convergence estimation error is proposed in this work and neither any analytic proof of the convergence of the estimation error is given, nor the time delay is taken into account. On the other hand, the state estimation in presence of delayed measurements is investigated in Orjuela et al. [2007]. Sufficient conditions, under a LMI form, are given for ensuring the state estimation convergence towards zero. However, the robust state estimation with respect to disturbances (e.g. noise) acting on the outputs has not been addressed in this work.

We introduce a so-called proportional-integral observer (PIO) which provides very interesting robustness properties with respect to perturbations [Beale and Shafai, 1989, Weinmann, 1991, Busawon and Kabore, 2001]. This observer is characterised by the use of two corrective injection terms, proportional and integral, instead of the conventional proportional correction frequently employed in the Luenberger observer and/or in its classical extensions. The PIO has also been successfully employed in the synchronization of a chaotic system by Hua and Guan [2005]. The extension of the PIO design, based on dissipativity framework, to a particular nonlinear system whose non linearity is assumed to satisfy a sector bounded constraint, has been recently proposed in Jung et al. [2007]. However, the PIO design for standard delayed linear system and for delayed multiple models (Takagi-Sugeno and/or decoupled multiple models) seems poorly investigated in the literature.

Contribution and paper organisation. In this communication, the PIO design for decoupled multiple models with delayed measurements is investigated. The outline of this paper is as follows. A brief description of the decoupled multiple model is presented in section 2 . The state estimation problem is presented in section 3 and sufficient conditions, on the basis of the Lyapunov-Krasovskii method, are established in order to ensure the stability of the PIO. Finally, in section 4, an academic example illustrates the state estimation of a decoupled multiple model.

Notations: The following standard notations will be used throughout the paper: $P>0(P<0)$ denotes a positive (negative) definite matrix $P ; X^{T}$ denotes the transpose of matrix $X$; I is the identity matrix of appropriate dimension and $\operatorname{diag}\{\}$ is a block diagonal matrix of appropriate dimension. The $\mathcal{L}_{2}-$ norm of a signal, quantifying its energy is denoted and defined by $\|e(t)\|_{2}^{2}=\int_{0}^{\infty} e^{T}(t) e(t) d t$.
We shall simply write $\mu_{i}(\xi(t))=\mu_{i}(t)$ and $x(t-\tau(t))=$ $x(\nabla)$ where $\tau(t)>0$ is a variable time-delay.

\section{DECOUPLED MULTIPLE MODEL}

The structure of the decoupled multiple model can be viewed as a parallel interconnection of several submodels via a weighted sum of their outputs [Gatzke and Doyle III, 1999]. In other words, as a particular class of hybrid heterogeneous models. By considering a state space representation, this structure takes the following form [Orjuela et al., 2008]:

$$
\begin{aligned}
\dot{x}_{i}(t) & =A_{i} x_{i}(t)+B_{i} u(t)+D_{i} w(t), \\
y_{i}(t) & =C_{i} x_{i}(t), \\
z(t) & =\sum_{i=1}^{L} \mu_{i}(\xi(t)) y_{i}(t), \\
y(t) & =z(t-\tau(t))+W w(t) .
\end{aligned}
$$

where $x_{i} \in \mathbb{R}^{n_{i}}$ and $y_{i} \in \mathbb{R}^{p}$ are respectively the state vector and the output of the $i^{\text {th }}$ submodel; $u \in \mathbb{R}^{m}$ is the multiple model input, $z \in \mathbb{R}^{p}$ is the multiple model output and $y \in \mathbb{R}^{p}$ the measured output subject to a variable time-delay $\tau(t)$ and a perturbation $w \in \mathbb{R}^{r}$. The matrices $A_{i} \in \mathbb{R}^{n_{i} \times n_{i}}, B_{i} \in \mathbb{R}^{n_{i} \times m}, D_{i} \in \mathbb{R}^{n_{i} \times r}, C_{i} \in \mathbb{R}^{p \times n_{i}}$ and $W \in \mathbb{R}^{p \times r}$ are known and constant.

Assumption 1. The perturbation is bounded energy signal, i.e. $\|w(t)\|_{2}^{2}<\infty$.

The variable time-delay $\tau(t)$ acting on the output is perfectly modelled by a continuous function that satisfies the following conditions [Wu et al., 2004]:

$$
\left\{\begin{array}{l}
0 \leq \tau(t) \leq \tau_{\max } \\
\dot{\tau}(t) \leq \beta<1
\end{array}\right.
$$

where $\tau_{\max }$ and $\beta$ are constant. This time-delay can be due for example to the inherent nature of the system, the sensor reactivity or the transmission data time-delay.

The relative contribution of each submodel to the global model according to the current operating point of the system is quantified by the weighting functions $\mu_{i}(\xi(t))$ (i.e. the transition law). They are associated to each operating zone and satisfy the following convex sum properties:

$$
\sum_{i=1}^{L} \mu_{i}(\xi(t))=1 \text { and } 0 \leq \mu_{i}(\xi(t)) \leq 1, \forall i=1 \ldots L, \forall t .
$$

The current operating point is taken into account by means of the so-called decision variable $\xi(t)$ which is a characteristic variable of the system (e.g. inputs and/or measured variables).

In this multiple model the contribution of each submodel is taken into account via a weighted sum of the submodel outputs. Consequently, heterogeneous submodels (i.e. submodels of different dimensions) can be considered in the modelling stage as a supplementary degree of flexibility and generality. Therefore, this structure is well suited for modelling nonlinear hybrid systems whose structure varies with the operating regime, for example when the complexity of the dynamic behaviour is not uniform across the operating space [Gregorcic and Lightbody, 2008]. 
Notice that the multiple model approach is an efficient way to deal with hybrid systems: no assumption is made on the continuity of the weighting functions blending the outputs of the submodels. Then switches between submodels can be modelled by discontinuous weighting functions taking boolean values in the set $[0,1]$.

\section{STATE ESTIMATION PROBLEM}

In a previous work [Orjuela et al., 2007], the state estimation of a decoupled multiple model subject to delayed measurements has been investigated on the basis of a proportional observer given by :

$$
\begin{aligned}
\dot{\hat{x}}_{i}(t) & =A_{i} \hat{x}_{i}(t)+B_{i} u(t)+K_{i}(y(t)-\hat{y}(t)), \\
\hat{z}(t) & =\sum_{i=1}^{L} \mu_{i}(\xi(t)) C_{i} \hat{x}_{i}(t) \\
\hat{y}(t) & =\hat{z}(t-\tau(t)),
\end{aligned}
$$

where $K_{i} \in \mathbb{R}^{n_{i} \times p}$ is the gain associated to $i^{\text {th }}$ observer. Sufficient conditions for ensuring the exponential convergence towards zero of the estimation errors $e_{i}(t)=$ $x_{i}(t)-\hat{x}_{i}(t)$ have been established. Note however that disturbances acting on the systems are not taken into consideration. Consequently, the sensitivity problem of the state estimation with respect to disturbances has not been previously addressed in this work. On the other hand, the proportional observer offers only one degree of freedom $K_{i}$ for reducing the influence of the disturbance and providing at the same time good dynamic performances (two antagonist design goals).

In this paper, the proportional observer (4) is replaced by a more general observer, known as proportional-integral observer (PIO), which provides very interesting robustness properties with respect to perturbations. In particular, good trade-off between dynamic and robust performances of the observer can be obtained with the PIO because two degrees of freedom are available for the observer design.

\subsection{Preliminaries}

For the simplicity of manipulations, the decoupled multiple model (1) is rewritten in the following compact form [Orjuela et al., 2007, 2008]:

$$
\begin{aligned}
\dot{x}(t) & =\tilde{A} x(t)+\tilde{B} u(t)+\tilde{D} w(t), \\
z(t) & =\tilde{C}(t) x(t) \\
y(t) & =z(\nabla)+W w(t)
\end{aligned}
$$

where:

$$
\begin{aligned}
x(t) & =\left[x_{1}^{T}(t) \cdots x_{i}^{T}(t) \cdots x_{L}^{T}(t)\right]^{T} \in \mathbb{R}^{n}, n=\sum_{i=1}^{L} n_{i}, \\
\tilde{A} & =\operatorname{diag}\left\{A_{1} \cdots A_{i} \cdots A_{L}\right\} \in \mathbb{R}^{(n \times n)}, \\
\tilde{B} & =\left[B_{1}^{T} \cdots B_{i}^{T} \cdots B_{L}^{T}\right]^{T} \in \mathbb{R}^{(n \times m)}, \\
\tilde{D} & =\left[D_{1}^{T} \cdots D_{i}^{T} \cdots D_{L}^{T}\right]^{T} \in \mathbb{R}^{(n \times r)}, \\
\tilde{C}(t) & =\left[\mu_{1}(t) C_{1} \cdots \mu_{i}(t) C_{i} \cdots \mu_{L}(t) C_{L}\right] \in \mathbb{R}^{(p \times n)} .
\end{aligned}
$$

The matrix $\tilde{C}(t)$ is time-varying because the weighting functions are taken into consideration in this matrix.

\subsection{PIO structure}

In a PIO, the classic used proportional action given by the gains $K_{i}$ in (4) is replaced by the use of two correction actions: proportional and integral. For this purpose, a supplementary integral variable $y_{I}(t)=\int_{0}^{t} y(\eta) d \eta$ must be taken into consideration in the PIO architecture. Note however that this integration can be replaced by a more general action, for example, by considering the filtered output signal as follows:

$$
\dot{y}_{I}(t)=N y_{I}(t)+y(t)
$$

where the matrix $N$ is a fading effect coefficient matrix that regulates the transient response of $y_{I}(t)$ [Jung et al., 2007].

Let us notice that the equations (5) and (7) can be gathered as follows:

$$
\begin{aligned}
\dot{x}_{a}(t) & =\left(\bar{C}_{1} \tilde{A} \bar{C}_{1}^{T}+\bar{C}_{2} N \bar{C}_{2}^{T}\right) x_{a}(t)+\bar{C}_{2} \tilde{C}(\nabla) \bar{C}_{1}^{T} x_{a}(\nabla) \\
& +\bar{C}_{1} \tilde{B} u(t)+\left(\bar{C}_{2} W+\bar{C}_{1} \tilde{D}\right) w(t), \\
y(t) & =\tilde{C}(\nabla) \bar{C}_{1}^{T} x_{a}(\nabla)+W w(t), \\
y_{I}(t) & =\bar{C}_{2}^{T} x_{a}(t)
\end{aligned}
$$

where the augmented vector $x_{a} \in \mathbb{R}^{(n+p)}$ and the constant bloc matrices $\bar{C}_{1}$ and $\bar{C}_{2}$ of appropriate dimensions are respectively given by:

$$
x_{a}(t)=\left[\begin{array}{c}
x(t) \\
y_{I}(t)
\end{array}\right], \bar{C}_{1}=\left[\begin{array}{c}
\mathrm{I}_{(n \times n)} \\
0_{(p \times n)}
\end{array}\right], \bar{C}_{2}=\left[\begin{array}{c}
0_{(n \times p)} \\
\mathrm{I}_{(p \times p)}
\end{array}\right] .
$$

The structure of the proposed PIO can be obtained on the basis of the augmented form (8):

$$
\begin{aligned}
& \dot{\hat{x}}_{a}(t)=\left(\bar{C}_{1} \tilde{A} \bar{C}_{1}^{T}+\bar{C}_{2} N \bar{C}_{2}^{T}\right) \hat{x}_{a}(t)+\bar{C}_{2} \tilde{C}(\nabla) \bar{C}_{1}^{T} \hat{x}_{a}(\nabla) \\
& +\tilde{K}_{P}(y(t)-\hat{y}(t))+\tilde{K}_{I}\left(y_{I}(t)-\hat{y}_{I}(t)\right)+\bar{C}_{1} \tilde{B} u(t),(10 \mathrm{a}) \\
& \hat{y}(t)=\tilde{C}(\nabla) \bar{C}_{1}^{T} \hat{x}_{a}(\nabla), \\
& \hat{y}_{I}(t)=\bar{C}_{2}^{T} \hat{x}_{a}(t) .
\end{aligned}
$$

where the gains $\tilde{K}_{P}$ and $\tilde{K}_{I}$ must be designed and the matrices $\tilde{A}, \tilde{B}$ and $\tilde{C}(t)$ have been previously defined in (6). Let us notice that an additional injection term, given by the filtered (or integral) output estimation error $y_{I}(t)-\hat{y}_{I}(t)$, is included in the dynamic equation of the observer. Hence some degrees of freedom are in this way introduced for observer design. The use of the two gains $\tilde{K}_{P}$ (proportional action) and $\tilde{K}_{I}$ (integral action) is at the origin of the name Proportional-Integral Observer.

\subsection{Problem formulation}

The dynamics of the estimation error

$$
e_{a}(t)=x_{a}(t)-\hat{x}_{a}(t)
$$

can be easily established by considering the gathered forms of the decoupled multiple model (8) and the PIO (10): 


$$
\dot{e}_{a}(t)=\Lambda_{1} e_{a}(t)+\Lambda_{2}(\nabla) e_{a}(\nabla)+\Lambda_{3} w(t),
$$

where

$$
\begin{aligned}
\Lambda_{1} & =\bar{C}_{1} \tilde{A} \bar{C}_{1}^{T}+\bar{C}_{2} N \bar{C}_{2}^{T}-K_{I} \bar{C}_{2}^{T}, \\
\Lambda_{2}(\nabla) & =\left(\bar{C}_{2}-K_{P}\right) \tilde{C}(\nabla) \bar{C}_{1}^{T}, \\
\Lambda_{3} & =\left(\bar{C}_{2}-K_{P}\right) W+\bar{C}_{1} \tilde{D} .
\end{aligned}
$$

Let us remark, from equations (13a) and (13c), that the proportional gain $K_{P}$ can be used to modulate the influence of the disturbances on the estimation error $e_{a}(t)$ and the observer dynamics can be improved with the help of the integral gain $K_{I}$.

Remark 1. Note, from equation (13c), that the disturbance $w(t)$ can be totally decoupled (i.e. its influence is null) if and only if $K_{P} W=\bar{C}_{2} W+\bar{C}_{1} \tilde{D}$. Notice that

$$
\left[\begin{array}{c}
\tilde{D} \\
W
\end{array}\right]=K_{P} W=\left[\begin{array}{c}
K_{P 1} \\
\mathrm{I}
\end{array}\right] W
$$

and consequently, $K_{P 1}=\tilde{D} W^{T}\left(W W^{T}\right)^{-1}$. So, this condition cannot be easily satisfied in practice because $W$ is not necessary of complete rank. Besides, the total disturbance decoupling case reduce inevitably the freedom degrees to adjust the dynamic performances.

The robust PIO design problem can thus be formulated as finding the matrices $\tilde{K}_{P}$ and $\tilde{K}_{I}$ such that the influence of $w(t)$ on the estimation error (11) is attenuated and the state estimation error remains globally bounded for any blend between the submodels. To this end, the following objective signal $\nu(t)$ which only depends on the estimation error $e_{a}(t)$ is introduced:

$$
\nu(t)=H e_{a}(t),
$$

where $H$ is a matrix of appropriate dimension chosen by the designer. The weighting matrix $H$ in (15) can be selected for totally or partially taking into consideration the transfer from $w(t)$ to $e_{a}(t)$.

Finally, the expected performances of the PIO can be formulated by the following robust performances:

$$
\begin{aligned}
& \lim _{t \rightarrow \infty} e_{a}(t)=0 \text { for } w(t)=0, \\
& \|\nu(t)\|_{2}^{2} \leq \gamma^{2}\|w(t)\|_{2}^{2} \text { for } w(t) \neq 0 \text { and } \nu(0)=0,
\end{aligned}
$$

where $\gamma$ is the $\mathcal{L}_{2}$ gain from $w(t)$ to $\nu(t)$ to be minimized. Notice that condition (16a) ensures convergence towards zero of the estimation error in the perturbation free case. On the other hand, condition (16b) guarantees attenuation level on the estimation error with respect to perturbations. In the following, we will investigate the exponential convergence of the estimation error. Indeed, the exponential convergence is a strong form of convergence that guarantees dynamic performances of the estimation error, in particular a convergence velocity via a decay rate.

\subsection{Computation of the gains $\tilde{K}_{P}$ and $\tilde{K}_{I}$}

This section focuses on the computation of the PIO gains $\tilde{K}_{P}$ and $\tilde{K}_{I}$. For this purpose, the Lyapunov-Krasovskii functional method is employed in order to provide delayindependent sufficient conditions, in LMIs terms [Boyd et al., 1994], for ensuring the robust performances (16).

Theorem 1. Consider the decoupled multiple model (1) with time-delay (2) and assumption 1. There exists a PIO (10) ensuring the objectives (16) if there exists two symmetric, positive definite matrices $P$ and $Q$, two matrices $G_{P}$ and $G_{I}$ and a positive scalar $\bar{\gamma}$ solution of the constrained optimisation problem:

$$
\begin{aligned}
& \min \bar{\gamma} \quad \text { subject to } \\
& {\left[\begin{array}{ccc}
H^{T} H+\Lambda_{1}^{T} P+P \Lambda_{1}+Q & \tilde{\Lambda}_{2, i} & \Lambda_{3} \\
\tilde{\Lambda}_{2, i}^{T} & -(1-\beta) e^{-2 \alpha \tau_{\max }} Q & 0 \\
\Lambda_{3}^{T} & 0 & -\bar{\gamma} \mathrm{I}
\end{array}\right]<0}
\end{aligned}
$$

for $i=1 \cdots L$ where

$$
\begin{aligned}
& \Lambda_{1}=\bar{C}_{1} \tilde{A} \bar{C}_{1}^{T}+\bar{C}_{2} N \bar{C}_{2}^{T}+\alpha \mathrm{I}-\mathrm{G}_{\mathrm{I}} \overline{\mathrm{C}}_{2}^{\mathrm{T}}, \\
& \tilde{\Lambda}_{2, i}=\left(P \bar{C}_{2}-G_{P}\right) \tilde{C}_{i} \bar{C}_{1}^{T} \text {, } \\
& \Lambda_{3}=\left(P \bar{C}_{2}-G_{P}\right) W+P \bar{C}_{1} \tilde{D}, \\
& \tilde{C}_{i}=\left[\begin{array}{lllll}
0 & \cdots & C_{i} & \cdots & 0
\end{array}\right] .
\end{aligned}
$$

for a given decay rate $\alpha$ and for prescribed matrices $H$ and $N$. The observer gains are given by $K_{P}=P^{-1} G_{P}$ and $K_{I}=P^{-1} G_{I}$; the $\mathcal{L}_{2}$ gain from $w(t)$ to $\nu(t)$ is given by $\gamma=\sqrt{\bar{\gamma}}$.

Proof. The exponential convergence of the estimation error (11) is investigated via a Lyapunov-Krasovskii functional proposed by Mondié and Kharitonov [2005]:

$$
V(t)=e_{a}^{T}(t) P e_{a}(t)+\int_{-\tau(t)}^{0} e_{a}^{T}(t+\theta) e^{2 \alpha \theta} Q e_{a}(t+\theta) d \theta
$$

where $P$ and $Q$ are symmetric, positive definite matrices. Mondié and Kharitonov [2005] propose this functional in order to provide sufficient delay-independent conditions for the exponential stability of linear systems with constant time-delay. We use the same functional but by considering a time-varying delay. On the other hand, the Lyapunov-Krasovskii functional (17) can be rewritten as follows:

$$
\begin{aligned}
V(t) & =\left[\begin{array}{c}
e_{a}(t) \\
e_{a}(\nabla)
\end{array}\right]^{T}\left[\begin{array}{ll}
P & 0 \\
0 & 0
\end{array}\right]\left[\begin{array}{c}
e_{a}(t) \\
e_{a}(\nabla)
\end{array}\right] \\
& +\int_{-\tau(t)}^{0} e_{a}^{T}(t+\theta) e^{2 \alpha \theta} Q e_{a}(t+\theta) d \theta .
\end{aligned}
$$

On the other hand, the robust state estimation objectives (16) are guaranteed if there exists a Lyapunov-Krasovskii functional (17) such that [Boyd et al., 1994]:

$$
\dot{V}(t)+2 \alpha V(t)<\gamma^{2} w^{T}(t) w(t)-\nu^{T}(t) \nu(t)
$$

where $\alpha$ is the so-called decay rate for convergence velocity and $\gamma$ is the attenuation level from $w(t)$ to $\nu(t)$ for robust estimation. So, inequality (19) must be ensured in order to provide a solution of the robust state estimation problem. 
Let us consider initially the time-derivative of the functional (17) evaluated via Leibniz-Newton formula:

$$
\begin{aligned}
\dot{V}(t) & =\dot{e}_{a}^{T}(t) P e_{a}(t)+e_{a}^{T}(t) P \dot{e}_{a}(t)+e_{a}^{T}(t) Q e_{a}(t) \\
& -(1-\dot{\tau}(t)) e^{-2 \alpha \tau(t)} e_{a}^{T}(\nabla) Q e_{a}(\nabla) \\
& -2 \alpha \int_{-\tau(t)}^{0} e_{a}^{T}(t+\theta) e^{2 \alpha \theta} Q e_{a}(t+\theta) d \theta
\end{aligned}
$$

that can be upper bounded, using the a priori knowledge (2) of the time-delay as follows:

$$
\begin{aligned}
\dot{V}(t) & \leq \dot{e}_{a}^{T}(t) P e_{a}(t)+e_{a}^{T}(t) P \dot{e}_{a}(t)+e_{a}^{T}(t) Q e_{a}(t) \\
& -(1-\beta) e^{-2 \alpha \tau_{\max }} e_{a}^{T}(\nabla) Q e_{a}(\nabla) \\
& -2 \alpha \int_{-\tau(t)}^{0} e_{a}^{T}(t+\theta) e^{2 \alpha \theta} Q e_{a}(t+\theta) d \theta
\end{aligned}
$$

which becomes finally by considering the dynamics of the estimation error (12):

$$
\begin{gathered}
\dot{V}(t) \leq-2 \alpha \int_{-\tau(t)}^{0} e^{T}(t+\theta) e^{2 \alpha \theta} Q e(t+\theta) d \theta \\
+w^{T}(t) \Lambda_{3}^{T} P e_{a}(t)+e_{a}^{T}(t) P \Lambda_{3} w(t) \\
+\left[\begin{array}{c}
e_{a}(t) \\
e_{a}(\nabla)
\end{array}\right]^{T}\left[\begin{array}{cc}
\Lambda_{1}^{T} P+P \Lambda_{1}+Q & P \Lambda_{2}(\nabla) \\
\Lambda_{2}^{T}(\nabla) P & -(1-\beta) e^{-2 \alpha \tau_{\max } Q}
\end{array}\right]\left[\begin{array}{c}
e_{a}(t) \\
e_{a}(\nabla)
\end{array}\right] .
\end{gathered}
$$

Hence, the left-hand side of the inequality (19) is ensured by the following inequality:

$$
\begin{gathered}
\dot{V}+2 \alpha V \leq\left[\begin{array}{c}
e_{a}(t) \\
e_{a}(\nabla) \\
w(t)
\end{array}\right]^{T}\left\{\left[\begin{array}{ccc}
2 \alpha P & 0 & P \Lambda_{3} \\
0 & 0 & 0 \\
\Lambda_{3}^{T} P & 0 & 0
\end{array}\right]\right. \\
\left.+\left[\begin{array}{ccc}
\Lambda_{1}^{T} P+P \Lambda_{1}+Q & P \Lambda_{2}(\nabla) & 0 \\
\Lambda_{2}^{T}(\nabla) P & -(1-\beta) e^{-2 \alpha \tau_{\max }} & 0 \\
0 & 0 & 0
\end{array}\right]\right\}\left[\begin{array}{c}
e_{a}(t) \\
e_{a}(\nabla) \\
w(t)
\end{array}\right] .
\end{gathered}
$$

Now, by considering (23) then the inequality (19) can be guaranteed if the following inequality holds:

$$
\begin{aligned}
& {\left[\begin{array}{c}
e_{a}(t) \\
e_{a}(\nabla) \\
w(t)
\end{array}\right]^{T}\left\{\left[\begin{array}{ccc}
\Lambda_{1}^{T} P+P \Lambda_{1}+Q & P \Lambda_{2}(\nabla) & 0 \\
\Lambda_{2}^{T}(\nabla) P & -(1-\beta) e^{-2 \alpha \tau_{\max }} & 0 \\
0 & 0 & 0
\end{array}\right]\right.} \\
& \left.\quad+\left[\begin{array}{ccc}
2 \alpha P+H^{T} H & 0 & P \Lambda_{3} \\
0 & 0 & 0 \\
\Lambda_{3}^{T} P & 0 & -\gamma^{2} \mathrm{I}
\end{array}\right]\right\}\left[\begin{array}{c}
e_{a}(t) \\
e_{a}(\nabla) \\
w(t)
\end{array}\right]<0 .
\end{aligned}
$$

Let us notice that the above inequality is a quadratic form in $\left[e_{a}(t) e_{a}(\nabla) w(t)\right]^{T}$. Consequently, the inequality (19) for ensuring robust objectives (16) is finally guaranteed if the following inequality holds:

$$
\left[\begin{array}{ccc}
2 \alpha P+H^{T} H+\Lambda_{1}^{T} P+P \Lambda_{1}+Q & P \Lambda_{2}(\nabla) & P \Lambda_{3} \\
\Lambda_{2}^{T}(\nabla) P & -(1-\beta) e^{-2 \alpha \tau_{\max }} Q & 0 \\
\Lambda_{3}^{T} P & 0 & -\gamma^{2} \mathrm{I}
\end{array}\right]<0
$$

Note that a time-varying matrix $\Lambda_{2}(\nabla)$, given by (13b), appears in this inequality and its resolution cannot be computed using standard LMI algorithms.
However, the time-delayed varying matrix $\tilde{C}(\nabla)$ in $\Lambda_{2}(\nabla)$ can be rewritten as a weighted sum of constant matrices as follows:

$$
\tilde{C}(\nabla)=\sum_{i=1}^{L} \mu_{i}(\nabla) \tilde{C}_{i}
$$

where $\tilde{C}_{i}$ is a constant block matrix given by:

$$
\tilde{C}_{i}=\left[\begin{array}{lllll}
0 & \cdots & C_{i} & \cdots & 0
\end{array}\right]
$$

such that the term $C_{i}$ is found on the $i^{\text {th }}$ block column of $\tilde{C}_{i}$. By considering (26) then the inequality (25) becomes

$\sum_{i=1}^{L} \mu_{i}(\nabla)\left[\begin{array}{ccc}2 \alpha P+H^{T} H+\tilde{\Lambda}_{1}^{T} P+P \tilde{\Lambda}_{1}+Q & P \tilde{\Lambda}_{2, i} & P \Lambda_{3} \\ \tilde{\Lambda}_{2, i}^{T} P & -(1-\beta) e^{-2 \alpha \tau_{\max }} Q & 0 \\ \Lambda_{3}^{T} P & 0 & -\gamma^{2} \mathrm{I}\end{array}\right]<0$.

where the constant matrices $\tilde{\Lambda}_{2, i}$ are given by:

$$
\tilde{\Lambda}_{2, i}=\left(\bar{C}_{2}-K_{P}\right) \tilde{C}_{i} \bar{C}_{1}^{T} .
$$

According to the properties (3) of the weighting functions, the above matrix inequality (28) can be satisfied by considering the simultaneous solution of the set of inequalities expressed for each upper bound of $\mu_{i}(\nabla)$. On the other hand, the linearisation of the matrix inequality (28) can be achieved by the standard change of variables $G_{P}=P \tilde{K}_{P}$, $G_{I}=P \tilde{K}_{I}$ and $\gamma^{2}=\bar{\gamma}$. Hence, sufficient conditions for ensuring the robust performances (16) are obtained under a LMI form which can be solved via classical LMI tools. The proof of theorem 1 is completed.

\section{SIMULATION EXAMPLE}

Let us consider the state estimation of a hybrid heterogeneous multiple models with $L=2$ submodels. The parameters of the submodels are:

$$
\begin{array}{rlrl}
A_{1} & =\left[\begin{array}{ccc}
-2 & 0.5 & 0.6 \\
-0.3 & -0.9 & 0 \\
-1.3 & 0.6 & -0.8
\end{array}\right], & A_{2}=\left[\begin{array}{cc}
-0.2 & -0.6 \\
0.3 & -1
\end{array}\right], \\
B_{1}=\left[\begin{array}{lll}
1 & 0.2 & 0.5
\end{array}\right]^{T}, & B_{2}=\left[\begin{array}{cc}
-0.5 & 0.8
\end{array}\right]^{T}, \\
D_{1}=\left[\begin{array}{ccc}
0.1 & 0.2 & -0.3
\end{array}\right]^{T}, & D_{2}=\left[\begin{array}{cc}
-0.1 & 0.1
\end{array}\right]^{T}, \\
C_{1}=\left[\begin{array}{ccc}
1 & 0.8 & 0.5 \\
0.2 & 0.3 & -0.5
\end{array}\right], & C_{2}=\left[\begin{array}{cc}
0.7 & 0.3 \\
0.2 & -0.5
\end{array}\right], \\
W & =\left[\begin{array}{ll}
0.2 & -0.3
\end{array}\right]^{T} . &
\end{array}
$$

The time-delay function appearing in the output of the systems is given by $\tau(t)=0.5+0.45 \sin (0.5 t)$ with $\tau_{\max }=$ 0.95. The upper bound of its derivative is $\beta=0.225$. Here, the decision variable $\xi(t)$ is the input signal $u(t) \in[0,1]$. The weighting functions are obtained from normalised Gaussian functions:

$$
\begin{aligned}
& \mu_{i}(\xi(t))=\omega_{i}(\xi(t)) / \sum_{j=1}^{L} \omega_{j}(\xi(t)), \\
& \omega_{i}(\xi(t))=\exp \left(-\left(\xi(t)-c_{i}\right)^{2} / \sigma^{2}\right),
\end{aligned}
$$

with $\sigma=0.4, c_{1}=0.3$ and $c_{2}=0.7$.

A solution that satisfies the theorem 1 , is given by: 


$$
\begin{aligned}
\tilde{K}_{P} & =\left[\begin{array}{ccccccc}
0.075 & 0.144 & -0.220 & -0.074 & 0.075 & 0.999 & 0.002 \\
-0.237 & -0.444 & 0.678 & 0.225 & -0.238 & 0.002 & 0.992
\end{array}\right]^{T}, \\
\tilde{K}_{I} & =\left[\begin{array}{lllllll}
-0.431 & 0.139 & -0.068 & -0.054 & 0.046 & 2.269 & -0.045 \\
-0.141 & -0.434 & -0.114 & -0.022 & 0.011 & 0.042 & 2.252
\end{array}\right]^{T},
\end{aligned}
$$

with a decay rate $\alpha=0.2$ and an attenuation level $\gamma^{2}=0.5$ and for $H=\mathrm{I}$ and $N=-0.3$.

In the simulation the disturbance acting on the output is a normally distributed random signal. The state estimation error is plotted in figure 1 (left) where $e_{i}$ is the $i^{\text {th }}$ component of the estimation error vector (11). The measured and the estimated outputs are shown in figure 1 (right). In both cases, the error around the origin time is due to the differences between initial conditions of the multiple model and the observer.
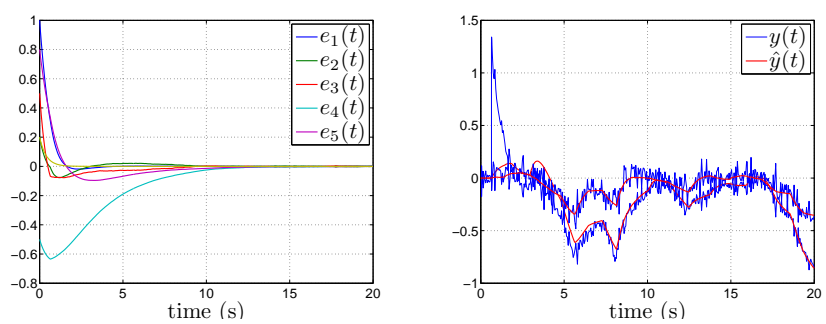

Figure 1. State estimation errors (left). Actual and estimated outputs (right).

\section{CONCLUSION}

A proportional-integral observer (PIO), on the basis of a decoupled multiple model, is proposed in this contribution in order to cope with the state estimation problem of a nonlinear hybrid system in presence of delayed and perturbed measurements. The decoupled multiple model is an interesting structure for modelling nonlinear systems with variable structure because the dimensions of the employed submodels can be different. On the other hand, the PIO offers more degree of freedom for robust state estimation with respect to the classic proportional observer previously proposed. The robust stability problem of the estimation error is investigated using the Lyapunov-Krasovskii functional method and delay-independent sufficient conditions, under a LMI form, are established.

Further research will be to investigate the state estimation by considering distributed delays in the submodel dynamic equations.

\section{REFERENCES}

R. Babuska. Fuzzy Modeling for Control. Kluwer Academic Publishers, London, 1998.

S. Beale and B. Shafai. Robust control system design with a proportional integral observer. International Journal of Control, 50(1):97-111, 1989.

P. Bliman. LMI characterization of the strong delayindependent stability of linear delay systems via quadratic Lyapunov-Krasovskii functionals. Systems and Control Letters, 43(4):263-274, 2001.

A. Boukhris, G. Mourot, and J. Ragot. Non-linear dynamic system identification: a multiple-model approach. International Journal of Control, 72(7/8):591604, 1999.
S. Boyd, L. El Ghaoui, E. Feron, and V. Balakrishnan. Linear Matrix Inequalities in System and Control Theory. SIAM Studies in Applied Mathematics 15, Philadelphia, 1994.

K.K. Busawon and P. Kabore. Disturbance attenuation using proportional integral observers. International Journal of Control, 74(74):618-627, 2001.

D. Filev. Fuzzy modeling of complex systems. International Journal of Approximate Reasoning, 5(3):281-290, 1991.

E. P. Gatzke and F. J. Doyle III. Multiple model approach for CSTR control. In 14th IFAC World Congress, Beijing, China, 1999.

G. Gregorcic and G. Lightbody. Nonlinear system identification: From multiple-model networks to gaussian processes. Engineering Applications of Artificial Intelligence, 21(7):1035-1055, 2008.

C. Hua and X. Guan. Synchronization of chaotic systems based on PI observer design. Physics Letters A, 334 (5-6):382-389, 2005.

J. Jung, K. Huh, and T. Shim. Dissipative proportional integral observer for a class of uncertain nonlinear systems. In American Control Conference, ACC, New York City, USA, 2007.

C. Lin, Q.G. Wang, T.H. Lee, and Y. He. LMI Approach to Analysis and Control of Takagi-Sugeno Fuzzy Systems with Time Delay. Springer-Verlag Berlin Heidelberg, 2007.

S. Mondié and V.L. Kharitonov. Exponential estimates for retarded time-delay systems: An LMI approach. IEEE Transactions on Automatic Control, 50(2):268273, 2005.

R. Murray-Smith and T.A. Johansen. Multiple model Approaches to Modelling and Control. Taylor \& Francis, London, 1997.

R. Orjuela, B. Marx, D. Maquin, and J. Ragot. A decoupled multiple model approach for state estimation of nonlinear systems subject to delayed measurements. In 3rd IFAC Advanced Fuzzy and Neural Network Workshop, AFNC, Valenciennes, France, 2007.

R. Orjuela, B. Marx, D. Maquin, and J. Ragot. State estimation for nonlinear systems using a decoupled multiple mode. International Journal of Modelling Identification and Control, 4(1):59-67, 2008.

J.P. Richard. Time-delay systems: an overview of some recent advances and open problems. Automatica, 39(10): 1667-1694, 2003.

O. Sename. New trends in design of observers for timedelay systems. Kybernetika, 37(4):427-458, 2001.

C.A. Smith and A.B. Corripio. Principles and practice of automatic process control. John Wiley \& Sons, 1985.

K. Tanaka and H. Wang. Fuzzy Control Systems design and analysis. John Wiley \& Sons, New York, 2001.

F.J. Uppal, R.J. Patton, and M. Witczak. A neuro-fuzzy multiple-model observer approach to robust fault diagnosis based on the DAMADICS benchmark problem. Control Engineering Practice, 14(6):699-717, 2006.

A. Weinmann. Uncertain Models and Robust Control. Springer-Verlag Wien New York, Vienne, 1991.

M. Wu, Y. He, J.H. She, and G.P. Liu. Delay-dependent criteria for robust stability of time-varying delay systems. Automatica, 40(8):1435-1439, 2004. 